\title{
DIVERGENCE POINTS OF DEFORMED EMPIRICAL MEASURES
}

\author{
L. Olsen
}

\begin{abstract}
We introduce and develope a unifying multifractal framework based on deformations of empirical measures. This framework (1) unifies and extends many results in multifractal analysis of local characteristics of dynamical systems and "fractal" measures and (2) provides a systematic basis for the detailed study of divergence points.
\end{abstract}

\section{Introduction and Statement of Results}

\subsection{Multifractal analysis.}

In the study of geometric properties of dynamical systems or "fractal" measures one is often interested in the asymptotic behaviour of various local quantities associated with the underlying dynamical or geometric structure, e.g. the ergodic average of a continuous function, the local entropy or the local Lyapunov exponent. These quantities provide a description of various aspects of dynamical systems, e.g. chaoticity, sensitive dependence, etc., and provide important information about the underlying dynamical and/or geometric structure.

Multifractal spectra. This idea leads to the notion of multifractal spectra. Let $X$ be a metric space and and let $Y$ be a set. Let $\varphi: M \rightarrow Y$ be a function defined on a subset $M$ of $X$. We now define the multifractal spectrum of $\varphi$ by

$$
\begin{aligned}
& f(t)=\operatorname{dim}\{x \in M \mid \varphi(x)=t\}, \quad t \in Y, \\
& F(t)=\operatorname{Dim}\{x \in M \mid \varphi(x)=t\}, \quad t \in Y
\end{aligned}
$$

where dim and Dim denote the Hausdorff dimension and the packing dimension, respectively. The multifractal spectra of various local characteristics $\varphi$ have recently been investigated, see for example [BSa, BSS, CM, FFW, Mo, Ols1, Ols2, Ols3, OW1, PW, PoW, TV1, TV2, W] and the references therein.

Received April 15, 2002.

2000 Mathematics Subject Classification. 37D35, 37D20, 28A80.

Key words and phrases. fractals, multifractals, multifractal spectrum, local dimensions, spectrum of local Lyapunov exponents, spectrum of local entropies, spectrum of ergodic averages, empirical measures, divergence points, self-conformal sets, self-conformal measures. 
Divergence points. Local characteristics of dynamical systems or "fractal" measures are typically defined in terms of limit processes, i.e. typically the local characteristics $\varphi: M \rightarrow \mathbb{R}$ has the form

$$
\varphi(x)=\lim _{n} \varphi_{n}(x)
$$

for a sequence of functions $\varphi_{n}: X \rightarrow \mathbb{R}$. For example, if $X=\Sigma$ is a subshift of finite type and $S: \Sigma \rightarrow \Sigma$ is the shift map, then the ergodic average of a continuous function $f: \Sigma \rightarrow \mathbb{R}$ at the point $\omega \in \Sigma$ is defined by the following limit $\lim _{n} \frac{1}{n} \sum_{k=0}^{n-1} f\left(S^{k} \omega\right)$. However, the limits involved do not necessarily exist at all points. Points for which the limits do not exist are called divergence points. The set of divergence points have until very recently been considered of little interest in dynamical systems and geometric measure theory. Indeed, according to folklore, these sets carried no essential information about the underlying structure. However, recent work [BSa, BSc, BSS, CX, FF, FFW, Ols2, Ols3, OW1, OW2, PW] has changed this point of view: typically the set of divergence points is extremely big, namely, it has full Hausdorff dimension, i.e.

$$
\operatorname{dim}\left\{x \in X \mid \text { the } \operatorname{limit} \lim _{n} \varphi_{n}(x) \text { does not exists }\right\}=\operatorname{dim} X .
$$

We introduce and develop a multifractal framework based on deformations of empirical measures. This framework unifies and extends many results in multifractal analysis of local characteristics of dynamical systems and "fractal" measures, and provides a systematic basis for the detailed study of divergence points. In particular, we emphasize the following novelties in this work:

- By considering particular deformations, we obtain essentially all multifractal spectra that have been studied, cf. Section 1.2.

- We do not limit ourselves to the study of the set divergence points, but provide a detailed study of the behaviour of individual divergence points. If $\mathrm{A}\left(\varphi_{n}(x)\right)$ denotes the set of accumulation points of the sequence $\left(\varphi_{n}(x)\right)_{n}$, then we compute the dimension of the set

$$
\left\{x \in X \mid T \subseteq \mathrm{A}\left(\varphi_{n}(x)\right) \subseteq S\right\}
$$

for $T, S \subseteq X$. This significantly generalizes the classes of sets that have been studied previously and has numerous applications, cf. Section 2

\subsection{Deformations of empirical measures.}

Let $(\mathrm{V}, \mathrm{E})$ be a finite directed graph where $\mathrm{V}$ denotes the set of vertices and $E$ denotes the set of edges. If $e \in E$, we denote the initial vertex of e by $i(e)$ and we denote the terminal vertex of e by $\mathrm{t}(\mathrm{e})$. For each vertex $i \in \mathrm{V}$, let $\Sigma_{i}$ denote the set of all infinite paths in (V,E) starting at $i$ and let $\Sigma$ denote the set of all infinite paths in $(\mathrm{V}, \mathrm{E})$, i.e.

$$
\begin{aligned}
\Sigma_{i} & =\left\{\mathrm{e}_{1} \mathrm{e}_{2} \mathrm{e}_{3} \ldots \mid \mathrm{e}_{k} \in \mathrm{E}, \mathrm{i}\left(\mathrm{e}_{1}\right)=i, \mathrm{t}\left(\mathrm{e}_{k}\right)=\mathrm{i}\left(\mathrm{e}_{k+1}\right)\right\} \\
\Sigma & =\left\{\mathrm{e}_{1} \mathrm{e}_{2} \mathrm{e}_{3} \ldots \mid \mathrm{e}_{k} \in \mathrm{E}, \mathrm{t}\left(\mathrm{e}_{k}\right)=\mathrm{i}\left(\mathrm{e}_{k+1}\right)\right\} .
\end{aligned}
$$


Also, let $S: \Sigma \rightarrow \Sigma$ denote the shift, $S\left(\mathrm{e}_{1} \mathrm{e}_{2} \mathrm{e}_{3} \ldots\right)=\mathrm{e}_{2} \mathrm{e}_{3} \mathrm{e}_{3} \ldots$ We denote the family of probability measures on $\Sigma$ by $\mathcal{P}(\Sigma)$. For a positive integer $n$, the $n$ 'th empirical measure $L_{n}: \Sigma \rightarrow \mathcal{P}(\Sigma)$ is defined by

$$
L_{n} \omega=\frac{1}{n} \sum_{k=0}^{n-1} \delta_{S^{k} \omega},
$$

where $\delta_{x}$ denotes the Dirac measure at $x$. We now consider continuous deformations of $L_{n}$, i.e. pairs $(X, \Xi)$ where $X$ is a metric space and $\Xi: \mathcal{P}(\Sigma) \rightarrow X$ is continuous with respect to the weak topology. We think of the composite

$$
\Xi \circ L_{n}: \Sigma \stackrel{L_{n}}{\longrightarrow} \mathcal{P}(\Sigma) \stackrel{\Xi}{\longrightarrow} X
$$

as a continuous deformation of the empirical measure $L_{n}$. For a sequence $\left(x_{n}\right)_{n}$ in a metric space $X$, we let $\mathrm{A}\left(x_{n}\right)$ denote the set of accumulation points of the sequence $\left(x_{n}\right)_{n}$, i.e.

$$
\mathrm{A}\left(x_{n}\right)=\left\{x \in X \mid \text { there exists a subsequence }\left(x_{n_{k}}\right)_{k} \text { such that } x_{n_{k}} \rightarrow x\right\} .
$$

For a deformation $(X, \Xi)$ and $T, S \subseteq X$, we define the multifractal decomposition set $\Delta_{i}(T, S)$ by

$$
\Delta_{i}(T, S)=\left\{\omega \in \Sigma_{i} \mid T \subseteq \mathrm{A}\left(\Xi L_{n} \omega\right) \subseteq S\right\},
$$

and we define the multifractal spectra $f_{i}(T, S)$ and $F_{i}(T, S)$ by

$$
\begin{aligned}
f_{i}(T, S) & =\operatorname{dim} \pi_{i} \Delta_{i}(T, S), \\
F_{i}(T, S) & =\operatorname{Dim} \pi_{i} \Delta_{i}(T, S),
\end{aligned}
$$

where $\pi_{i}: \Sigma_{i} \rightarrow \mathbb{R}^{d}$ denotes the projection map in (1.8). If $T=\varnothing$ and $S=\{t\}$ is a singleton, then the spectra $f_{i}(T, S)=f_{i}(\varnothing,\{t\})$ and $F_{i}(T, S)=F_{i}(\varnothing,\{t\})$ equal the classical multifractal spectra (1.1),

$$
\begin{aligned}
& f_{i}(\varnothing,\{t\})=\operatorname{dim} \pi_{i}\left\{\omega \in \Sigma_{i} \mid \lim _{n} \Xi L_{n} \omega=t\right\}, \\
& F_{i}(\varnothing,\{t\})=\operatorname{Dim} \pi_{i}\left\{\omega \in \Sigma_{i} \mid \lim _{n} \Xi L_{n} \omega=t\right\},
\end{aligned}
$$

and provide information about the points $\omega$ for which the $\operatorname{limit}_{n} \lim _{n} \Xi L_{n} \omega$ exists and equals $t$; however, if $T$ is non-empty and $S$ is not a singleton, then $f_{i}(T, S)$ and $F_{i}(T, S)$ provide detailed information about the distribution of individual divergence points. Section 1.3 contains a variational principle for $f_{i}(T, S)$ and $F_{i}(T, S)$. However, first we note that the notion of a deformation unifies most of the spectra that have been studied. Indeed, by choosing suitable deformations we obtain:

- The mixed spectrum of ergodic averages of arbitrary families of continuous functions. Let $\left(f_{j}\right)_{j \in I}$ be a (possible uncountable) family of continuous functions $f_{j}: \Sigma \rightarrow \mathbb{R}$ and assume that the family of maps $(\mathcal{P}(\Sigma) \rightarrow$ 
$\left.\mathbb{R}: \mu \rightarrow \int f_{j} d \mu\right)_{j \in I}$ is totally bounded. Define $\Xi: \mathcal{P}(\Sigma) \rightarrow \ell^{\infty}(I)$ by

$\Xi: \mu \rightarrow\left(\int f_{j} d \mu\right)_{j \in I}$. In this case we obtain for $T, S \subseteq \ell^{\infty}(I)$,

$$
\Delta_{i}(T, S)=\left\{\omega \in \Sigma_{i} \mid T \subseteq \mathrm{A}\left(\left(\frac{1}{n} \sum_{k=0}^{n-1} f_{j}\left(S^{k} \omega\right)\right)_{j \in I}\right) \subseteq S\right\} ;
$$

- Relative multifractal spectra, cf. Section 2.2;

- Mixed multifractal spectra, cf. Section 2.1 and Section 2.3.

In the examples below, $\left(K_{i}\right)_{i \in \mathrm{V}}$ and $\left(\mu_{i}\right)_{i \in \mathrm{V}}$ denote the self-conformal sets and self-conformal measures associated with a graph directed self-conformal iterated function system with probabilities $\left(\mathrm{V}, \mathrm{E},\left(V_{i}\right)_{i \in \mathrm{V}},\left(X_{i}\right)_{i \in \mathrm{V}},\left(S_{\mathrm{e}}\right)_{\mathrm{e} \in \mathrm{E}},\left(p_{\mathrm{e}}\right)_{\mathrm{e} \in \mathrm{E}}\right)$; details will be given in Section 1.3.

- The multifractal spectrum of $\mu_{i}$. Define $\Xi: \mathcal{P}(\Sigma) \rightarrow \mathbb{R}$ by $\Xi: \mu \rightarrow$

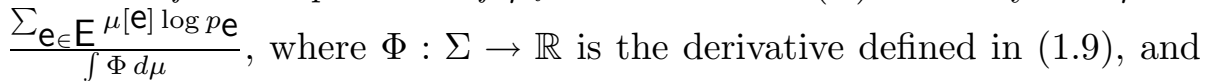
[e] is the cylinder generated by e. In this case we obtain for $T, S \subseteq \mathbb{R}$,

$$
\Delta_{i}(T, S)=\left\{\omega \in \Sigma_{i} \mid T \subseteq \mathrm{A}\left(\frac{\log \mu_{i} K_{\omega \mid n}}{\log \operatorname{diam} K_{\omega \mid n}}\right) \subseteq S\right\},
$$

where $K_{\omega \mid n}=S_{\mathrm{e}_{1}} \circ \cdots \circ S_{\mathrm{e}_{n}}\left(K_{\mathrm{t}\left(\mathrm{e}_{n}\right)}\right)$ for $\omega=\mathrm{e}_{1} \mathrm{e}_{2} \ldots \in \Sigma_{i}$;

- The spectrum of local entropies of $\mu_{i}$. Define $\Xi: \mathcal{P}(\Sigma) \rightarrow \mathbb{R}$ by $\Xi: \mu \rightarrow$ $\sum_{\mathrm{e} \in \mathrm{E}} \mu[\mathrm{e}] \log p_{\mathrm{e}}$, where [e] is the cylinder generated by e. In this case we obtain for $T, S \subseteq \mathbb{R}$,

$$
\Delta_{i}(T, S)=\left\{\omega \in \Sigma_{i} \mid T \subseteq \mathrm{A}\left(\frac{\log \mu_{i} K_{\omega \mid n}}{n}\right) \subseteq S\right\}
$$

where $K_{\omega \mid n}=S_{\mathrm{e}_{1}} \circ \cdots \circ S_{\mathrm{e}_{n}}\left(K_{\mathrm{t}\left(\mathrm{e}_{n}\right)}\right)$ for $\omega=\mathrm{e}_{1} \mathrm{e}_{2} \ldots \in \Sigma_{i}$

- The spectrum of local Lyapunov exponents. Define $\Xi: \mathcal{P}(\Sigma) \rightarrow \mathbb{R}$ by $\Xi: \mu \rightarrow \int \Phi d \mu$, where $\Phi: \Sigma \rightarrow \mathbb{R}$ is the derivative defined in (1.9). In this case we obtain for $T, S \subseteq \mathbb{R}$,

$$
\Delta_{i}(T, S)=\left\{\omega \in \Sigma_{i} \mid T \subseteq \mathrm{A}\left(\frac{\log \sup _{x}\left|\left(D S_{\omega \mid n}\right)(x)\right|}{n}\right) \subseteq S\right\},
$$

where $S_{\omega \mid n}=S_{\mathrm{e}_{1}} \circ \cdots \circ S_{\mathrm{e}_{n}}$ for $\omega=\mathrm{e}_{1} \mathrm{e}_{2} \ldots \in \Sigma_{i}$. $\left(\operatorname{Here}\left(D S_{\omega \mid n}\right)(x)\right.$ denotes the derivative of $S_{\omega \mid n}$ at $x$.)

\subsection{Statements of results.}

We first introduce the setting, viz. self-conformal sets and self-conformal measures. Let $\mathrm{E}_{i}$ denote the set of edges whose initial vertex equals $i$ and let $\mathrm{E}_{i j}$ denote the set of edges whose initial vertex equals $i$ and whose terminal vertex 
equals $j$. A graph directed conformal iterated function system with probabilities is a list

$$
\left(\mathrm{V}, \mathrm{E},\left(V_{i}\right)_{i \in \mathrm{V}},\left(X_{i}\right)_{i \in \mathrm{V}},\left(S_{\mathrm{e}}\right)_{\mathrm{e} \in \mathrm{E}},\left(p_{\mathrm{e}}\right)_{\mathrm{e} \in \mathrm{E}}\right)
$$

where

(1) $V_{i}$ is an open, connected subset of $\mathbb{R}^{d}$.

(2) $X_{i} \subseteq V_{i}$ is a compact set with $X_{i}^{\circ-}=X_{i}$.

(3) $S_{\mathrm{e}}: V_{j} \rightarrow V_{i}$ is a contractive $C^{1+\gamma}$ diffeomorphism with $0<\gamma<1$ such that $S_{\mathrm{e}}\left(X_{j}\right) \subseteq X_{i}$ for all $i, j \in \mathrm{V}$ and all e $\in \mathrm{E}_{i j}$.

(4) The Conformality Condition: $\left(D S_{\mathrm{e}}\right)(x)$ is a contractive similarity map. (Here $\left(D S_{\mathrm{e}}\right)(x)$ denotes the derivative of $S_{\mathrm{e}}$ at $x$.)

(5) $\left(p_{\mathrm{e}}\right)_{\mathrm{e} \in \mathrm{E}_{i}}$ is a probability vector for each $i \in \mathrm{V}$.

It follows from $[\mathrm{Hu}]$ that there exists a unique list $\left(K_{i}\right)_{i \in \mathrm{V}}$ of non-empty compact sets $K_{i} \subseteq X_{i}$ such that

$$
K_{i}=\bigcup_{\mathrm{e} \in E_{i}} S_{\mathrm{e}}\left(K_{\mathrm{t}(\mathrm{e})}\right)
$$

and a unique list $\left(\mu_{i}\right)_{i \in \mathrm{V}}$ of probability measures with supp $\mu_{i}=K_{i}$ such that

$$
\mu_{i}=\sum_{\mathrm{e} \in E_{i}} p_{\mathrm{e}} \mu_{\mathrm{t}(\mathrm{e})} \circ S_{\mathrm{e}}^{-1} .
$$

The sets $\left(K_{i}\right)_{i \in \mathrm{V}}$ and the measures $\left(\mu_{i}\right)_{i \in \mathrm{V}}$ are called the self-conformal sets and the self-conformal measures associated with the list (1.7), respectively. We will also assume that the so-called Strong Open Set condition (SOSC) is satisfied.

(6) The Strong Open Set Condition: There exists a list $\left(U_{i}\right)_{i \in \mathrm{V}}$ of open nonempty and bounded sets $U_{i} \subseteq X_{i}$ with $S_{\mathrm{e}}\left(U_{j}\right) \subseteq U_{i}$ for all $i, j \in \mathrm{V}$ and all e $\in \mathrm{E}_{i j}$ such that $S_{\mathrm{e}_{1}}\left(U_{\mathrm{t}\left(\mathrm{e}_{1}\right)}\right) \cap S_{\mathrm{e}_{2}}\left(U_{\mathrm{t}\left(\mathrm{e}_{2}\right)}\right)=\varnothing$ and $U_{i} \cap K_{i} \neq \varnothing$ for all $i \in \mathrm{V}$ and all $\mathrm{e}_{1}, \mathrm{e}_{2} \in \mathrm{E}_{i}$ with $\mathrm{e}_{1} \neq \mathrm{e}_{2}$.

For each vertex $i \in \mathrm{V}$ we define the projection $\pi_{i}: \Sigma_{i} \rightarrow K_{i}$ by

$$
\left\{\pi_{i} \omega\right\}=\bigcap_{n} S_{\mathrm{e}_{1}} \circ \cdots \circ S_{\mathrm{e}_{n}}\left(K_{\mathrm{t}\left(\mathrm{e}_{n}\right)}\right)
$$

for $\omega=\mathrm{e}_{1} \mathrm{e}_{2} \mathrm{e}_{3} \ldots \in \Sigma_{i}$. Finally define $\Phi: \Sigma \rightarrow \mathbb{R}$ by

$$
\Phi \omega=\log \left|\left(D S_{\mathrm{e}_{1}}\right)\left(\pi_{\mathrm{t}\left(\mathrm{e}_{1}\right)}(S \omega)\right)\right|
$$

for $\omega=\mathrm{e}_{1} \mathrm{e}_{2} \mathrm{e}_{3} \ldots \in \Sigma$. The map $\Phi$ represents the local change of scale as one goes from $\pi_{\mathrm{t}\left(\mathrm{e}_{1}\right)}(S \omega)$ to $\pi_{\mathrm{i}\left(\mathrm{e}_{1}\right)}(\omega)$. Let $\mathcal{P}_{S}(\Sigma)$ denote the family of shift invariant probability measures on $\Sigma$, and let $\mathcal{E}_{S}(\Sigma)$ denote the family of ergodic shift invariant probability measures on $\Sigma$. Finally, $h(\mu)$ denotes the entropy of the measure $\mu$.

We can now state the main results. The first result, Theorem 1.1, gives an upper and a lower bound for the multifractal spectrum $f_{i}(\varnothing, C)$ for an arbitrary continuous deformation $(X, \Xi)$. 
Theorem 1.1. The multifractal spectrum $f_{i}(\varnothing, C)$. Let $X$ be a metric space and let $\Xi: \mathcal{P}(\Sigma) \rightarrow X$ be a continuous map. If $C \subseteq X$ is closed, then

$$
\sup _{x \in C} \sup _{\substack{\mu \in \mathcal{E}_{S}(\Sigma) \\ \Xi \mu=x}}-\frac{h(\mu)}{\int \Phi d \mu} \leq f_{i}(\varnothing, C) \leq \sup _{x \in C} \sup _{\substack{\mu \in \mathcal{P}_{S}(\Sigma) \\ \Xi \mu=x}}-\frac{h(\mu)}{\int \Phi d \mu} .
$$

The second main result, Theorem 1.2, provides a variational principle for the general multifractal spectrum $f_{i}(T, S)$. If $X$ is a vector space and $d$ is a metric in $X$, then we will say that $d$ is linearly compatible if

(1) For all $x_{1}, x_{2}, y_{1}, y_{2} \in X$ we have $d\left(x_{1}+x_{2}, y_{1}+y_{2}\right) \leq d\left(x_{1}, y_{1}\right)+d\left(x_{2}, y_{2}\right)$.

(2) For all $x, y \in X$ and all $\lambda \in \mathbb{R}$ we have $d(\lambda x, \lambda y) \leq|\lambda| d(x, y)$.

Observe that if $d$ is induced by a norm, then $d$ is linearly compatible. Let $\mathcal{C}(X)=\{C \subseteq X \mid C$ is closed and convex $\}$ and equip $\mathcal{C}(X)$ with the Hausdorff metric. Finally, for a subset $T$ of $X$, we denote the closed convex hull of $T$ by $\overline{\mathrm{CO}}(T)$. We can now state Theorem 1.2 and Theorem 1.3.

Theorem 1.2. The multifractal spectra $f_{i}(T, S)$ and $F_{i}(T, S)$. Let $X$ be a vector space with a linearly compatible metric and let $\Xi: \mathcal{P}(\Sigma) \rightarrow X$ be a continuous affine map. Fix $T, S \subseteq \Xi(\mathcal{P}(\Sigma))$.

(1) If $T=\varnothing$ and $S$ is closed and convex, then

$$
f_{i}(T, S)=F_{i}(T, S)=\sup _{x \in S} \sup _{\substack{\mu \in \mathcal{P}_{S}(\Sigma) \\ \Xi \mu=x}}-\frac{h(\mu)}{\int \Phi d \mu}
$$

(2) If $T \neq \varnothing$ and $T$ is contained in a connected component of $S$, then

$$
\sup _{\substack{T \subseteq Q \subseteq S \\ T \text { is a continuum }}} \inf _{x \in Q} \sup _{\substack{\mu \in \mathcal{P}_{S}(\Sigma) \\ \Xi \mu=x}}-\frac{h(\mu)}{\int \Phi d \mu} \leq f_{i}(T, S) \leq \inf _{x \in T} \sup _{\substack{\mu \in \mathcal{P}_{S}(\Sigma) \\ \Xi \mu=x}}-\frac{h(\mu)}{\int \Phi d \mu} .
$$

(3) If $T \neq \varnothing$ and $\overline{\mathrm{co}}(T)$ is contained in a connected component of $S$, then

$$
f_{i}(T, S)=\inf _{x \in T} \sup _{\substack{\mu \in \mathcal{P}_{S}(\Sigma) \\ \Xi \mu=x}}-\frac{h(\mu)}{\int \Phi d \mu} .
$$

The variational principle in (3) is, in general, not true for the packing dimension spectrum $F_{i}(T, S)$, cf. the example below.

(4) If $T \neq \varnothing$ and $T$ is not contained in a connected component of $S$, then

$$
\left\{\omega \in \Sigma_{i} \mid T \subseteq \mathrm{A}\left(\Xi L_{n} \omega\right) \subseteq S\right\}=\varnothing .
$$


Example showing that the variational principle in Theorem 1.2.(3) is, in general, not true for the packing spectrum $F_{i}(T, S)$. Let $N$ be a positive integer with $N \geq 2$. For $x \in[0,1]$, let $x=\sum_{n=1}^{\infty} \frac{\varepsilon_{n}(x)}{N^{n}}$ denote the unique non-terminating $N$-adic expansion of $x$. Also, for $x \in[0,1]$ and a digit $i \in\{0,1, \ldots, N-1\}$, write $\Pi_{i}(x ; n)=\frac{\left|\left\{1 \leq k \leq n \mid \varepsilon_{k}(x)=i\right\}\right|}{n}$, i.e. $\Pi_{i}(x ; n)$ denotes the frequency of the digit $i$ among the first $n$ digits in the $N$-adic expansion of $x$, and let $\Pi(x ; n)=\left(\Pi_{i}(x ; n)\right)_{i=0,1, \ldots, N-1}$. Consider the case where the underlying graph $(\mathrm{V}, \mathrm{E})$ has only one vertex, $\star$ say, and $N$ edges labelled by $0,1, \ldots, N-1$. We define the sets $V_{\star}$ and $X_{\star}$ in $(1.7)$ by $V_{\star}=\mathbb{R}$ and $X_{\star}=[0,1]$, and we define the maps $S_{i}:[0,1] \rightarrow[0,1]$ by $S_{i}(x)=\frac{x+i}{N}$. Finally, we consider the deformation $\Xi: \mathcal{P}(\Sigma) \rightarrow \mathbb{R}^{N}$ given by $\Xi(\mu)=(\mu([i]))_{i=0,1, \ldots, N-1}$ where $[i]$ denotes the cylinder generated by $i$. For this choice of $\Xi$ we have

$$
\pi_{\star}\left(\Delta_{\star}(T, S)\right)=\{x \in[0,1] \mid T \subseteq \mathrm{A}(\Pi(x ; n)) \subseteq S\} .
$$

Letting $\Delta$ denote the simplex of probability vectors in $\mathbb{R}^{N}$, it therefore follows from Theorem 1.2.(3) that (see [Ols6] or [OW1] for details)

$$
\begin{aligned}
\operatorname{dim}\{x \in[0,1] \mid \mathrm{A}(\Pi(x ; n))=\Delta\} & =\operatorname{dim} \pi_{\star}\left(\Delta_{\star}(\Delta, \Delta)\right) \\
& =\inf _{\left(p_{0}, p_{1}, \ldots, p_{N-1}\right) \in \Delta} \sup _{\substack{\mu \in \mathcal{P}_{S}(\Sigma) \\
\mu([i])=p_{i}}} \frac{h(\mu)}{\log N} \\
& =0 .
\end{aligned}
$$

However, it follows from [Ols8] that the set $\{x \in[0,1] \mid \mathrm{A}(\Pi(x ; n))=\Delta\}$ is residual, whence

$$
\operatorname{Dim}\{x \in[0,1] \mid \mathrm{A}(\Pi(x ; n))=\Delta\}=1 .
$$

This shows that the variational principle in Theorem 1.2.(3) is, in general, not true for the packing spectrum.

Theorem 1.3. Upper semi-continuity of the multifractal spectrum $f_{i}(\varnothing, C)$. Let $X$ be a vector space with a linearly compatible metric and let $\Xi$ : $\mathcal{P}(\Sigma) \rightarrow X$ be a continuous affine map. Then the multifractal spectrum

$$
\mathcal{C}(X) \rightarrow \mathbb{R}: C \rightarrow f_{i}(\varnothing, C)
$$

is upper semi-continuous.

Theorem 1.1, Theorem 1.2.(1) and Theorem 1.3 are proved in [Ols2], and Theorem 1.2.(2) and Theorem 1.2.(3) are proved in [Ols3, OW2]. The proofs are based on techniques from large deviation theory and ergodic theory.

The variational principle in Theorem 1.2.(2) has many diverse and important applications, and the reader is referred to [Ols3, Ols5, Ols9, OW1, OW2] for a full list. Here we will just mention one of the most important and striking applications. For a large class of deformations $(X, \Xi)$ (including the deformations in (1.3), (1.4), (1.5) and (1.6)) Theorem 1.2.(3) implies that the set of divergence points, $\left\{\omega \in \Sigma_{i} \mid\right.$ the $\operatorname{limit}_{n} \lim _{n} \Xi L_{n} \omega$ does not exist $\}$, has full dimension. 
Theorem 1.4. The dichotomy theorem for divergence points. Let $X$ be a vector space with a linearly compatible metric and let $\Xi: \mathcal{P}(\Sigma) \rightarrow X$ be

a continuous affine map. Assume that the function $x \rightarrow \sup _{\substack{\mu \in \mathcal{P}_{S}(\Sigma) \\ \Xi \mu=x}}-\frac{h(\mu)}{\int \Phi d \mu}$ is continuous at the point where it attains its supremum.

(1) If $\Xi(\mathcal{P}(\Sigma))$ is a singleton, then

$$
\left\{\omega \in \Sigma_{i} \mid \text { the limit } \lim _{n} \Xi L_{n} \omega \text { does not exist }\right\}=\varnothing
$$

(2) If $\Xi(\mathcal{P}(\Sigma))$ is not a singleton, then

$$
\operatorname{dim} \pi_{i}\left\{\omega \in \Sigma_{i} \mid \text { the limit } \lim _{n} \Xi L_{n} \omega \text { does not exist }\right\}=\operatorname{dim} K_{i} .
$$

This results has previously only been obtained for local dimensions, ergodic averages, local entropies and local Luapunov exponents. However, Theorem 1.4 shows that the result are true for a far more general class of local characteristics, viz. all local characteristics defined in terms of deformations of empirical measures.

\section{Applications}

\subsection{An application: ergodic spectra.}

As an application of our main results, we will now state a variational principle for a very general type of mixed multifractal spectrum of ergodic averages. Mixed multifractal spectra combine different local characteristics which depend simultaneously on various different aspects of the underlying dynamical system; in this case the simultaneous behaviour of the functions belonging to some (possible uncountable) family $\left(f_{j}\right)_{j \in I}$ of functions. The result follows immediately by applying Theorem 1.2 and Theorem 1.4 to the deformation $\mu \rightarrow\left(\int f_{j} d \mu\right)_{j \in I}$.

Theorem 2.1. The mixed multifractal spectrum of ergodic averages of arbitrary families of continuous functions. Let $\left(f_{j}\right)_{j \in I}$ be a (possible uncountable) family of continuous functions $f_{j}: \Sigma \rightarrow \mathbb{R}$, and assume that the family of maps $\left(\mathcal{P}(\Sigma) \rightarrow \mathbb{R}: \mu \rightarrow \int f_{j} d \mu\right)_{j \in I}$ is totally bounded. Fix $T, S \subseteq$ $\ell^{\infty}(I)$.

(1) If $T=\varnothing$ and $S$ is closed and convex, then

$$
\begin{aligned}
\operatorname{dim} \pi_{i}\left\{\omega \in \Sigma_{i} \mid T \subseteq \mathrm{A}\left(\left(\frac{1}{n} \sum_{k=0}^{n-1} f_{j}\left(S^{k} \omega\right)\right)_{j \in I}\right) \subseteq S\right\} & =\sup _{x \in S} \sup _{\substack{\mu \in \mathcal{P}_{S}(\Sigma) \\
\int f_{j} d \mu=x \text { for all } j \in I}}-\frac{h(\mu)}{\int \Phi d \mu} .
\end{aligned}
$$


(2) If $T \neq \varnothing$ and $\overline{\mathrm{co}}(T)$ is contained in a connected component of $S$, then

$$
\begin{aligned}
& \operatorname{dim} \pi_{i}\left\{\omega \in \Sigma_{i} \mid T \subseteq \mathrm{A}\left(\left(\frac{1}{n} \sum_{k=0}^{n-1} f_{j}\left(S^{k} \omega\right)\right)_{j \in I}\right) \subseteq S\right\} \\
&=\inf _{x \in T} \sup _{\substack{\mu \in \mathcal{P}_{S}(\Sigma) \\
\int f_{j} d \mu=x \text { for all } j \in I}}-\frac{h(\mu)}{\int \Phi d \mu} .
\end{aligned}
$$

(3) If $T \neq \varnothing$ and $T$ is not contained in a connected component of $S$, then

$$
\left\{\omega \in \Sigma_{i} \mid T \subseteq \mathrm{A}\left(\left(\frac{1}{n} \sum_{k=0}^{n-1} f_{j}\left(S^{k} \omega\right)\right)_{j \in I}\right) \subseteq S\right\}=\varnothing .
$$

(4) If $\left\{\left(\int f_{j} d \mu\right)_{j \in I} \mid \mu \in \mathcal{P}(\Sigma)\right\}$ is a singleton, then

$$
\left\{\omega \in \Sigma_{i} \mid \text { the limit } \lim _{n}\left(\frac{1}{n} \sum_{k=0}^{n-1} f_{j}\left(S^{k} \omega\right)\right)_{j \in I} \text { does not exist }\right\}=\varnothing \text {. }
$$

If $\left\{\left(\int f_{j} d \mu\right)_{j \in I} \mid \mu \in \mathcal{P}(\Sigma)\right\}$ is not a singleton, then

$\operatorname{dim} \pi_{i}\left\{\omega \in \Sigma_{i} \mid\right.$ the limit $\lim _{n}\left(\frac{1}{n} \sum_{k=0}^{n-1} f_{j}\left(S^{k} \omega\right)\right)_{j \in I}$ does not exist $\}=\operatorname{dim} K_{i}$.

A few special cases of Theorem 2.1 have recently been investigated. The special case of Theorem 2.1.(1) for which $T=\varnothing, S$ equals a singleton and the family $\left(f_{j}\right)_{j \in I}$ is finite has recently been obtained by various authors [BSa, BSc, BSS, FF, FFW, Oli]. In fact, Fan, Feng \& Wu [FFW] and Olivier [Oli] considered the case in which the family $\left(f_{j}\right)_{j \in I}$ consisted of only one function. Also, Theorem 2.1.(4) has been obtained recently by Barreira \& Schmeling [BSc] and Fan, Feng \& Wu [FFW] for finite families $\left(f_{j}\right)_{j \in I}$ of continuous functions. We emphasize that the papers [BSa, BSc, BSS, FF, FFW, Oli] study the case for which $T=\varnothing$ and the set $S$ equals a singleton, and therefore do not contain information about the distribution of individual divergence points.

\subsection{An application: relative (or conditional) multifractal spectra.}

In many examples of interest the deformation map $\Xi: \mathcal{P}(\Sigma) \rightarrow X$ is the quotient between two affine maps, cf. example (1.4). This leads to an abstract notion of relative (or conditional) multifractal spectra. Let $X$ be an algebra equipped with a metric and let $\Xi_{1}, \Xi_{2}: \mathcal{P}(\Sigma) \rightarrow X$ be continuous maps with $\Xi_{2}(\mu) \neq 0$ for all $\mu \in \mathcal{P}(\Sigma)$. We define the multifractal spectrum of $\Xi_{1}$ relative to (or conditioned by) $\Xi_{2}$ by

$$
\operatorname{dim} \pi_{i}\left\{\omega \in \Sigma_{i} \mid \mathrm{A}\left(\frac{\Xi_{1} L_{n} \omega}{\Xi_{2} L_{n} \omega}\right) \subseteq C\right\}
$$

for $C \subseteq X$.

Of course, it follows immediately from Theorem 1.1 that the following lower and upper bounds for the relative spectrum hold. 
Theorem 2.2. Abstract relative multifractal spectra. Let $X$ be an algebra equipped with a metric and let $\Xi_{1}, \Xi_{2}: \mathcal{P}(\Sigma) \rightarrow X$ be continuous maps with $\Xi_{2}(\mu) \neq 0$ for all $\mu \in \mathcal{P}(\Sigma)$. Then

$$
\sup _{\substack{\mu \in \mathcal{E}_{S}(\Sigma) \\ \Xi_{1} \mu \\ \Xi_{1} \mu}}-\frac{h(\mu)}{\int \Phi d \mu} \leq \operatorname{dim} \pi_{i}\left\{\omega \in \Sigma_{i} \mid \mathrm{A}\left(\frac{\Xi_{1} L_{n} \omega}{\Xi_{2} L_{n} \omega}\right) \subseteq C\right\} \leq \sup _{\substack{\mu \in \mathcal{P}_{S}(\Sigma) \\ \Xi_{1} \mu \\ \Xi_{1} \mu}}-\frac{h(\mu)}{\int \Phi d \mu}
$$

for all closed subsets $C$ of $X$.

Several examples of relative multifractal spectra have been considered in the literature. Lévy Véhel \& Vojak [LVV] and Moran [Mo] have investigated relative multifractal spectra of local dimensions of self-similar measures, and Barreira \& Saussol [BSa] have studied relative multifractal spectra of ergodic averages.

Let $f, g: \Sigma \rightarrow \mathbb{R}$ be continuous with $g(\omega) \neq 0$ for all $\omega \in \Sigma$. The multifractal spectrum of the ergodic averages of $f$ relative to $g$ is defined by

$$
\operatorname{dim} \pi_{i}\left\{\omega \in \Sigma_{i} \mid \mathrm{A}\left(\frac{\sum_{k=0}^{n-1} f\left(S^{k} \omega\right)}{\sum_{k=0}^{n-1} g\left(S^{k} \omega\right)}\right) \subseteq C\right\}
$$

for $C \subseteq \mathbb{R}$. By letting $X=\mathbb{R}$ and defining $\Xi_{1}: \mathcal{P}(\Sigma) \rightarrow \mathbb{R}$ and $\Xi_{2}: \mathcal{P}(\Sigma) \rightarrow \mathbb{R}$ by $\Xi_{1} \mu=\int f d \mu$ and $\Xi_{2} \mu=\int g d \mu$ we immediately obtain the following result from Theorem 2.2.

Theorem 2.3. Let $f, g: \Sigma \rightarrow \mathbb{R}$ be continuous with $g(\omega) \neq 0$ for all $\omega \in \Sigma$. If $C \subseteq \mathbb{R}$ is closed, then

$$
\begin{aligned}
\sup _{\substack{\mu \in \mathcal{E}_{S}(\Sigma) \\
\frac{\int f d \mu}{\int g d \mu} \in C}}-\frac{h(\mu)}{\int \Phi d \mu} & \leq \operatorname{dim}_{i}\left\{\omega \in \Sigma_{i} \mid \mathrm{A}\left(\frac{\sum_{k=0}^{n-1} f\left(S^{k} \omega\right)}{\sum_{k=0}^{n-1} g\left(S^{k} \omega\right)}\right) \subseteq C\right\} \\
& \leq \sup _{\substack{\mu \in \mathcal{P}_{S}(\Sigma) \\
\frac{\int f d \mu}{\int g d \mu} \in C}}-\frac{h(\mu)}{\int \Phi d \mu} .
\end{aligned}
$$

We believe that equality holds in Theorem 2.3. If $f$ and $g$ satisfy a Hölder type condition and $C$ is singleton, then Barreira \& Saussol [BSa] have shown that equality holds in Theorem 2.3.

\subsection{An application: mixed multifractal spectra.}

Mixed multifractal spectra combine different local characteristics which depend simultaneously on various different aspects of the underlying dynamical system. This leads to an abstract notion of mixed multifractal spectra. Let $\left(X_{j}, \Xi_{j}\right)_{j \in I}$ be (a possible uncountable) family of deformations. We define the mixed multifractal spectrum of the family $\left(X_{j}, \Xi_{j}\right)_{j \in I}$ by

$$
\operatorname{dim} \pi_{i}\left\{\omega \in \Sigma_{i} \mid T \subseteq \mathrm{A}\left(\left(\Xi_{j} L_{n} \omega\right)_{j \in I}\right) \subseteq S\right\}
$$


for $T, S \subseteq \mathrm{X}_{j} X_{j}$. The mixed multifractal spectrum of the family $\left(X_{j}, \Xi_{j}\right)_{j \in I}$ combine information provided by the different deformations $\left(X_{j}, \Xi_{j}\right)$ and study how these depend on each other. The following variational principle for an abstract mixed multifractal spectrum follows immediately from Theorem 1.2Theorem 1.4.

Theorem 2.4. Abstract mixed multifractal spectra. Let $\left(X_{j}, \Xi_{j}\right)_{j \in I}$ be (a possible uncountable) family of deformations and assume that $X_{j}$ is a normed vector space and that $\Xi_{j}: \mathcal{P}(\Sigma) \rightarrow X_{j}$ is affine and continuous. Define the vector space $\left[\mathbf{X}_{j} X_{j}\right]^{\infty}$ by $\left[\mathbf{X}_{j} X_{j}\right]^{\infty}=\left\{\left(x_{j}\right)_{j} \in \mathbf{X}_{j} X_{j} \mid \sup _{j}\left\|x_{j}\right\|<\infty\right\}$, and equip $\left[\mathrm{X}_{j} X_{j}\right]^{\infty}$ with the norm $\left\|\left(x_{j}\right)_{j}\right\|=\sup _{j}\left\|x_{j}\right\|$. Assume that $\sup _{\mu \in \mathcal{P}(\Sigma), j}\left\|\Xi_{j} \mu\right\|<$ $\infty$ and that the map $\mathcal{P}(\Sigma) \rightarrow\left[\mathbf{X}_{j} X_{j}\right]^{\infty}: \mu \rightarrow\left(\Xi_{j} \mu\right)_{j}$ is continuous. Fix $T, S \subseteq\left[\mathrm{X}_{j} X_{j}\right]^{\infty}$.

(1) If $T=\varnothing$ and If $S$ is closed and convex, then

$$
\operatorname{dim} \pi_{i}\left\{\omega \in \Sigma_{i} \mid T \subseteq \mathrm{A}\left(\left(\Xi_{j} L_{n} \omega\right)_{j \in I}\right) \subseteq S\right\}=\sup _{x \in S} \sup _{\substack{\mu \in \mathcal{P}_{S}(\Sigma) \\ \Xi_{j}(\Sigma=x \text { for all } j \in I}}-\frac{h(\mu)}{\int \Phi d \mu} .
$$

(2) If $T \neq \varnothing$ and $\overline{\mathrm{co}}(T)$ is contained in a connected component of $S$, then

$$
\operatorname{dim} \pi_{i}\left\{\omega \in \Sigma_{i} \mid T \subseteq \mathrm{A}\left(\left(\Xi_{j} L_{n} \omega\right)_{j \in I}\right) \subseteq S\right\}=\inf _{x \in T} \sup _{\substack{\mu \in \mathcal{P}_{S}(\Sigma) \\ \Xi_{j} \mu=x \text { for all } j \in I}}-\frac{h(\mu)}{\int \Phi d \mu}
$$

(3) If $T \neq \varnothing$ and $T$ is not contained in a connected component of $S$, then

$$
\left\{\omega \in \Sigma_{i} \mid T \subseteq \mathrm{A}\left(\left(\Xi_{j} L_{n} \omega\right)_{j \in I}\right) \subseteq S\right\}=\varnothing .
$$

(4) Assume that each map $x \rightarrow \sup _{\substack{\mu \in \mathcal{P}_{S}(\Sigma) \\ \Xi_{j} \mu=x}} \frac{h(\mu)}{\int \Phi d \mu}$ is continuous at the point where it attains its maximum. If $\left\{\left(\Xi_{j} \mu\right)_{j \in I} \mid \mu \in \mathcal{P}(\Sigma)\right\}$ is a singleton, then

$$
\left\{\omega \in \Sigma_{i} \mid \text { the limit } \lim _{n}\left(\Xi_{j} L_{n} \omega\right)_{j \in I} \text { does not exist }\right\}=\varnothing .
$$

If $\left\{\left(\Xi_{j} \mu\right)_{j \in I} \mid \mu \in \mathcal{P}(\Sigma)\right\}$ is not a singleton, then

$$
\operatorname{dim} \pi_{i}\left\{\omega \in \Sigma_{i} \mid \text { the limit } \lim _{n}\left(\Xi_{j} L_{n} \omega\right)_{j \in I} \text { does not exist }\right\}=\operatorname{dim} K_{i} .
$$

Applying Theorem 2.4 to the deformations $\left(X_{j}, \Xi_{j}\right)_{j \in I}$ where $X_{j}=\mathbb{R}$ and $\Xi_{j}: \mathcal{P}(\Sigma) \rightarrow \mathbb{R}$ is defined by $\Xi_{j} \mu=\int f_{j} d \mu$ gives the mixed multifractal spectrum of ergodic averages in Theorem 2.1. By considering other deformations we obtain variational principles for other mixed multifractal spectra. For example, by 
applying Theorem 2.4 to the deformations in (1.5) and (1.6), we obtain the following variational principle for the mixed multifractal spectrum for the local entropy and the local Lyapunov exponent. If $C \subseteq \mathbb{R}^{2}$ is closed and convex, then

$$
\operatorname{dim} \pi_{i}\left\{\omega \in \Sigma_{i} \mid \mathrm{A}\left(\frac{1}{n} \log \mu_{i} K_{\omega \mid n}, \frac{1}{n} \log \sup _{x}\left|\left(D S_{\omega \mid n}\right)(x)\right|\right) \subseteq C\right\}
$$

$$
\begin{aligned}
& =\sup _{x \in C} \quad \sup _{\mu \in \mathcal{P}_{S}(\Sigma)}-\frac{h(\mu)}{\int \Phi d \mu} . \\
& \left(\sum_{\mathbf{e}_{\in} \mathbf{E}} \mu[\mathrm{e}] \log p_{\mathbf{e}}, \int \Phi d \mu\right)=x
\end{aligned}
$$

The special case of (2.1) for which the set $C$ is a singleton, i.e. $C=\{\alpha, \beta\}$ for $\alpha, \beta \in \mathbb{R}$, has been obtained by Barreira, Saussol \& Schmeling [BSS] in a somewhat more general setting.

\subsection{An application: number theory.}

The techniques developed in [Ols2, Ols3, Ols4, Ols5, OW1, OW2] can also be used to study the Hausdorff dimension of several new classes of sets of numbers defined in terms of the asymptotic behaviour of the frequencies of digits in their $N$-adic expansion. In particular, we emphasize that we obtain the Hausdorff dimension for a new and very large class of sets of numbers $x$ for which the limiting frequencies of digits do not exist. In addition to computing the Hausdorff dimension of several new classes of sets of numbers, our techniques can also be used to obtain simple proofs of (substantial generalizations of) known results on the Hausdorff dimension of sets of normal and non-normal numbers. For example, in [Ols6, OW1, OW2] we obtain generalizations of results by Cajar [Ca] on the Hausdorff dimension of sets of numbers with prescribed strings of digits and of the results by Drobot \& Turner [DB] on the Hausdorff dimension of sets of numbers with prescribed sums of digits. In fact, the detailed theory of multifractal divergence points developed in [Ols2, Ols3, OW1] is essential for addressing these problems. The reader is referred to [Ols5, Ols6, Ols7, Ols8] for details and further applications.

\section{References}

[BSa] L. Barreira, B. Saussol, Variational principles and mixed multifractal spectra, Trans. Amer. Math. Soc. 353 (2001), 3919-3944.

[BSc] L. Barreira, J. Schmeling, Sets of "non-typical" points have full topological entropy and full Hausdorff dimension, Israel J. Math. 116 (2000), 29-70.

[BSS] L. Barreira, B. Saussol, J. Schmeling, Higher-dimensional multifractal analysis, to appear in J. Math. Pures Appl.

[Ca] H. Cajar, Billingsley dimension in probability spaces, Lecture Notes in Mathematics, 892. Springer-Verlag, Berlin-New York, 1981.

[CM] R. Cawley, R. D. Mauldin, Multifractal decomposition of Moran fractals, Adv. Math. 92 (1992), 196-236.

[CX] E. Chen, J. Xiong, The pointwise dimension of self-similar measures, Chinese Sci. Bull. 44 (1999), 2136-2140. 
[DT] V. Drobot, J. Turner, Hausdorff dimension and Perron-Frobenius theory, Illinois J. Math. 33 (1989), 1-9.

[FF] A.-H. Fan, D.-J. Feng, On the distribution of long-term time averages on symbolic space, J. Statist. Phys. 99 (2000), 813-856.

[FFW] A.-H. Fan, D.-J. Feng, J. Wu, Recurrence, dimension and entropy, J. London Math. Soc. (2) 64 (2001), 229-244.

[Hu] J. Hutchinson, Fractals and self-similarity, Indiana Univ. Math. J. 30 (1981), 713747.

[LVV] J. Lévy Véhel, R. Vojak, Multifractal analysis of Choquet capacities, Adv. in Appl. Math. 20 (1998), 1-43.

[Mo] M. Moran, Problems on self-similar geometry, Fractal geometry and stochastics, II (Greifswald/Koserow, 1998), 69-93, Progr. Probab. 46, Birkhäuser, Basel, 2000.

[Oli] E. Olivier, Analyse multifractale de fonctions continues, C. R. Acad. Sci. Paris Sér. I Math. 326 (1998), 1171-1174.

[Ols1] L. Olsen, Multifractal geometry, Fractal geometry and stochastics, II (Greifswald/ Koserow, 1998), 3-37, Progr. Probab. 46, Birkhäuser, Basel, 2000.

[Ols2] _ Multifractal analysis of divergence points of deformed measure theoretical Birkhoff averages, preprint, 2001.

[Ols3] _ Multifractal analysis of divergence points of deformed measure theoretical Birkhoff averages. III, preprint, 2001.

[Ols4]_ Small sets of divergence points are dimensionless, preprint, 2001.

[Ols5] - Distribution of digits in integers: Besicovitch-Eggleston subsets of $\mathbb{N}$, preprint, 2001.

[Ols6] Dimensions of some sets of numbers defined by their $N$-adic expansion, to appear in Math. Proc. Cambridge Philos. Soc.

[Ols7] _ Distribution of digits in integers: fractal dimensions and zeta functions, to appear in Acta Arithmetica.

[Ols8] , Extremely non-normal numbers, preprint, 2002.

[Ols9] , Mixed divergence points for self-similar measures, preprint, 2002.

[OW1] L. Olsen, S. Winter, Normal and non-normal points of self-similar sets and divergence points of self-similar measures, to appear in Jour. London Math. Soc.

[OW2] Multifractal analysis of divergence points of deformed measure theoretical Birkhoff averages. II, preprint, 2001.

[PW] Y. Pesin, H. Weiss, The multifractal analysis of Birkhoff averages and large deviations, Global analysis of dynamical systems, 419-431, Inst. Phys., Bristol, 2001.

[PoW] M. Pollicott, H. Weiss, Multifractal analysis of Lyapunov exponent for continued fraction and Manneville-Pomeau transformations and applications to Diophantine approximation, Comm. Math. Phys. 207 (1999), 145-171.

[TV1] F. Takens, E. Verbitski, Multifractal analysis of local entropies for expansive homeomorphisms with specification, Comm. Math. Phys. 203 (1999), 593-612.

[TV2] - General multifractal analysis of local entropies, Fund. Math. 165 (2000), 203-237.

[We] H. Weiss, The Lyapunov spectrum for conformal expanding maps and axiom-A surface diffeomorphisms, J. Statist. Phys. 95 (1999), 615-632.

Department of Mathematics, University of St. Andrews, St. Andrews, Fife KY16 9SS, SCOTLAND.

E-mail address: lo@st-and.ac.uk 